\title{
Excreción de nitrógeno amoniacal total a diferentes densidades de siembra de Cyprinus carpio en condiciones de laboratorio
}

\section{Total ammonia nitrogen excretion at different Cyprinus carpio sowing densities in laboratory conditions}

\section{Excreção de nitrogênio amoniacal total em diferentes densidades de Cyprinus carpio em condições de laboratório}

\author{
Ana C. Torres-Mesa ${ }^{1 *}$; Mario O. Tovar-Bohórquez ${ }^{2 *}$; Hernán Hurtado-Giraldo ${ }^{3}$; \\ Edwin Gómez-Ramírez ${ }^{*}$ \\ 1 BSc, \\ $2 \mathrm{CPhD}$, \\ $3 \mathrm{PhD}$, \\ $4 \mathrm{MSc}$ \\ * Grupo de investigación en Ictiología, Facultad de Ciencias Básicas, Universidad Militar Nueva Granada, Cajicá, Colombia. \\ Email: edwin.gomez@gmail.com
}

Recibido: marzo 11 de 2013. Aceptado: mayo 22 de 2015.

\begin{abstract}
Resumen
En sistemas acuícolas la excreción de nitrógeno amoniacal total (NAT) está determinada por varios parámetros biológicos y fisicoquímicos como la densidad de siembra. Si no hay un adecuado control de NAT el consumo de alimento y el crecimiento disminuye eventualmente conduciendo a la muerte. Por lo tanto, definir la relación entre la densidad de siembra y la tasa de excreción de NAT, es importante para establecer las condiciones adecuadas para el crecimiento. En el presente trabajo se utilizó como modelo experimental la carpa común (Cyprinus carpio). Se evaluaron dos tratamientos: T1, 30 individuos y T2, 60 individuos por unidad experimental (acuarios de $40 \mathrm{l}$ ) con tres repeticiones. Los cuales fueron medidos (peso, longitud total y estándar) al inicio y cada 14 días. Semanalmente se realizó seguimiento de $\mathrm{pH}$, temperatura, dureza de carbonatos y general. Para el registro del NAT y oxígeno disuelto, se tomaron muestras cada cuatro días. La tasa de excreción de NAT fue calculada por periodos de 14 días. Los resultados obtenidos para la tasa de excreción de NAT fueron: el día 14 para el T1=0.69 $\pm 0.009 \mu \mathrm{g} \mathrm{g}^{-1}$ hora $^{-1}$, con una biomasa de $18.33 \pm 0.13 \mathrm{~g}$; para el T2 $=0.40 \pm 0.02 \mu \mathrm{g} \mathrm{g}^{-1} \mathrm{hora}^{-1} \mathrm{y}$ biomasa de $34.66 \pm 0.33 \mathrm{~g}$; el 28 para el T1=0.53 $\pm 0.02 \mu \mathrm{g} \mathrm{g}^{-1}$ hora $^{-1}$, con una biomasa de $24.8 \pm 0.3 \mathrm{~g}$; para el $\mathrm{T} 2=0.28 \pm 0.03$

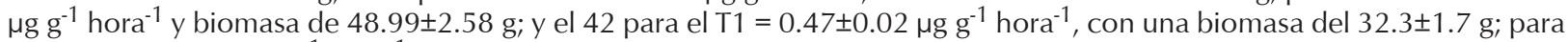
el T2 $=0.26 \pm 0.02 \mu \mathrm{g} \mathrm{g}^{-1}$ hora $^{-1}$ y biomasa de $69.81 \pm 7.04 \mathrm{~g}$. Después de estos resultados se puede concluir que a mayor densidad de siembra la tasa de excreción de amonio total por gramo disminuye.
\end{abstract}

Palabras clave: calidad del agua, osmorregulación, biomasa, mecanismos de excreción.

\begin{abstract}
Total ammonia-nitrogen (TAN) excretion from aquaculture systems is determined by several biological and physicochemical parameters, such as sowing density. If TAN is not suitably controlled, food consumption and growth will become reduced,
\end{abstract}


eventually leading to death. The relationship between sowing density and TAN excretion rate must thus be defined for establishing suitable conditions for fish growth. The common carp (Cyprinus carpio) was used as experimental model in this work. Two treatments were evaluated (T1 involved 30 individuals and T2 60 individuals per experimental unit in 40 I aquariums) with three repeats; this involved measuring weight and total and standard length at the start of the experiment and every 14 days thereafter. Weekly follow-up was made regarding water $\mathrm{pH}$, temperature and carbonate and overall hardness. Samples were taken every four days for recording TAN and dissolved oxygen. The TAN excretion rate was calculated for 14 day periods. TAN excretion rate result on day 14 for T1 was $0.69 \pm 0.009 \mu g^{-1}$ hour $^{-1}$ with $18.33 \pm 0.13 \mathrm{~g}$ biomass and $0.40 \pm 0.02 \mathrm{mg} \mathrm{g}^{-1}$ hour- ${ }^{1}$ with $34.66 \pm 0.33 \mathrm{~g}$ biomass for T2; on day $28 \mathrm{~T} 1$ gave $0.53 \pm 0.02 \mathrm{\mu g} \mathrm{g}^{-1} \mathrm{hour}^{-1}$ with $24.8 \pm 0.3 \mathrm{~g}$ biomass and $0.28 \pm 0.03 \mu \mathrm{g} \mathrm{g}^{-1}$ hour $^{-1}$ and $48.99 \pm 2.58 \mathrm{~g}$ biomass for T2; on day $42 \mathrm{~T} 1=0.47 \pm 0.02 \mu \mathrm{g} \mathrm{g}^{-1} \mathrm{hour}^{-1}$ with $32.3 \pm 1.7 \mathrm{~g}$ biomass and $\mathrm{T} 2=0.26 \pm 0.02 \mathrm{\mu g} \mathrm{g}^{-1}$ hour $^{-1}$ with $69.81 \pm 7.04 \mathrm{~g}$ biomass. Such results led to concluding that the total ammonia excretion per gram rate decreased with higher sowing density.

Key words: water quality, osmoregulation, biomass, excretion mechanism.

\section{Resumo}

Em sistemas de aquicultura a excreção de nitrogênio amoniacal total (NAT) é determinada por vários parâmetros biológicos e físico-químicos tais como a densidade de estocagem. Se não há um adequado controle de NAT, o consumo de alimento e o crescimento diminuem, eventualmente levando à morte. definir a relação entre a densidade de estocagem e a taxa excreção de NAT é importante para estabelecer as condições ideais para o crescimento. No presente trabalho foi utilizada a carpa comum (Cyprinus carpio) como um modelo experimental. Dois tratamentos foram avaliados: T1, 30 indivíduos e T2, 60 indivíduos/ unidade experimental (aquários de 40 litros) com três repetições. Os peixes foram medidos (peso, comprimento total e padrão) no início e cada 14 dias. Foram medidos semanalmente pH, temperatura, dureza de carbonatos e geral. Para o registro da NAT e oxigênio dissolvido, as amostragens foram feitas a cada quatro dias. A taxa de excreção de NAT foi calculada em períodos de 14 dias. A taxa de excreção de NAT foi: dia 14 para T1 $=0.69 \pm 0.009 \mu^{-1}$ hora $^{-1}$, com uma biomassa de $18.33 \pm 0.13 \mathrm{~g}$; para T2 $=0.40 \pm 0.02 \mu \mathrm{g} \mathrm{g}^{-1}$ hora $^{-1}$ e biomassa de $34.66 \pm 0.33 \mathrm{~g}$; dia 28 para T1 $=0.53 \pm 0.02 \mu \mathrm{g} \mathrm{g}{ }^{-1} \mathrm{hora}^{-1}, \mathrm{com}^{\circ}$ uma biomassa de $24.8 \pm 0.3 \mathrm{~g}$; para $\mathrm{T} 2=0.28 \pm 0.03 \mu \mathrm{g} \mathrm{g}^{-1}$ hora $^{-1}$ e biomassa de $48.99 \pm 2.58 \mathrm{~g}$; e dia 42 para $\mathrm{T} 1=0.47 \pm 0.02 \mu \mathrm{g}$ $\mathrm{g}^{-1}$ hora $^{-1}$, com uma biomassa de 32.3 $\pm 1.7 \mathrm{~g}$; para T2 $=0.26 \pm 0.02 \mu \mathrm{g} \mathrm{g}^{-1}$ hora $^{-1}$ e biomassa de $69.81 \pm 7.04$ g. Pode-se concluir que a taxa de excreção de amônio total por grama diminui o aumento da densidade de estocagem.

Palavras-chave: qualidade da água, osmorregulação, biomassa, mecanismos de excreção.

\section{Introducción}

En Colombia, Cyprinus carpio es una especie introducida, a partir de la década de los 40`s, distribuyéndose en todos los pisos térmicos adquiriendo gran importancia comercial en algunas localidades (Valderrama et al., 2004).

En teleósteos la excreción de desechos nitrogenados, se produce principalmente en forma de amonio no ionizado $\left(\mathrm{NH}_{3}\right)$, resultado del catabolismo de aminoácidos, denominándolos organismos amoniotélicos (Walsh y Mommsen, 2001; Remen et al., 2008), además en peces, el nitrógeno amoniacal total (NAT) es el primer producto metabólico, representado entre el 70 al 95\% del nitrógeno total excretado (Kaushik, 2000).

El $\mathrm{NH}_{3}$ es una molécula altamente tóxica (Walsh y Mommsem, 2001; Ip, et al., 2001; Hargreaves y Tucker, 2004), por lo tanto debe ser rápidamente eliminado de la sangre hacia el ambiente a través de las branquias (Nelson y Cox, 2004).

En peces de agua dulce, la excreción de $\mathrm{NH}_{3}$ tiene lugar por difusión pasiva y/o transporte facilitado (glicoproteínas Rhesus), por medio de gradientes favorables de presión parcial entre la sangre y el agua
(Rombough, 2011), facilitada por la acidificación de la sangre, la alta solubilidad del $\mathrm{NH}_{3}$ y a través del mucus secretado por las branquias (Ip et al., 2001; Wood, 2001; Remen et al., 2008; Ip y Chew, 2010). En sistemas cerrados de cultivo o con bajo flujo de agua, a medida que la concentración de NAT en el medio externo aumenta, la eliminación a través de las branquias disminuye debido a que las diferencias entre los gradientes de presión parcial entre la sangre y el agua tienden a ser iguales (Rombough, 2011).

Cuando los gradientes de concentración de $\mathrm{NH}_{3}$ comienzan a ser tóxicos porque la tasa de producción supera a la tasa de eliminación, se activa otro sistema de excreción mediado por las ATPasas, a través de un intercambio entre el ión sodio $\left(\mathrm{Na}^{+}\right)$y el ión amonio $\left(\mathrm{NH}_{4}^{+}\right)$(Wood, 2001), el cual mantiene un equilibrio del NAT dentro y fuera del pez, disminuyendo así la toxicidad del $\mathrm{NH}_{3}$ (Ip, et al., 2004).

En la acuicultura, la concentración de $\mathrm{NH}_{3}$ excretada por los organismos depende de varios factores, como: el hábito alimenticio (Ballantyne, 2001), el porcentaje de proteína en la comida (Wood, 2001; Mercado et al., 2006), la ración de alimentación, la temperatura del agua (Wood, 2001), el oxígeno disuelto (Remen et 
al., 2008), el pH (Scott et al., 2005), la biomasa (ReddyLopata et al., 2006), la edad de los individuos (Papoutsoglou et al., 1987) y la densidad de siembra (Biswas et al., 2006).

En cuanto a este último factor, se ha determinado que influye negativamente en el bienestar, la sobrevivencia, el crecimiento y producción de peces (Rahman et al., 2005; Chakraborty y Mirza, 2007), por lo tanto es importante determinar la densidad de siembra para encontrar una adecuada relación entre el buen crecimiento de los peces y la ganancia económica (Ip y Chew, 2010).

Por tal motivo, este trabajo pretende definir la dinámica de excreción de nitrógeno amoniacal total (NAT) en función de la densidad de siembra en alevinos de C. carpio; teniendo en cuenta la importancia de estos dos factores principalmente en sistemas cerrados de cultivo. Por ejemplo en sistema de recirculación, evaluar dicha dinámica ayuda a definir la capacidad de carga para estimar las tasas de biofiltración necesaria para remover este producto metabólico de desecho. En el caso de los sistemas acuapónicos la misma información puede ser utilizada para definir la densidad de plantas a producir por unidad de área dependiendo de la edad y densidad de peces. Por último, esta investigación contribuirá a estimar la descarga de nitrógeno de los sistemas acuícolas tradicionales hacia los efluentes que generalmente son cuerpos de agua naturales.

\section{Materiales y métodos}

Este trabajo fue realizado en el Laboratorio de Fisiología Animal en la sede Campus Cajicá, de la Universidad Militar Nueva Granada, ubicada longitud oeste $74^{\circ} 02^{\prime}$, latitud norte $4^{\circ} 55^{\prime}$ (datum WGS 84) y altitud de $2580 \mathrm{msnm}$, con una temperatura promedio anual entre $12-18^{\circ} \mathrm{C}$ y humedad relativa del $88 \%$ (González et al., 2009; Gobernación de Cundinamarca, 2011).

\section{Material biológico}

Para la realización de este proyecto, se adquirieron 300 alevinos de carpa común (C. carpio) con un peso promedio de $0.58 \pm 0.12 \mathrm{~g}$, los cuales se manipularon teniendo en cuenta las normas éticas para el manejo de peces (CCAC, 2005). Durante una semana los peces se mantuvieron en dos acuarios de $40 \mathrm{l}$, se alimentaron ad libitum tres veces al día con alimento comercial Truchina ${ }^{\circledR}$ al $45 \%$ de proteína, en este tiempo estuvieron bajo inspección constante para establecer la condición de salud de los especímenes (Nado erráti$\mathrm{co}$, presencia de patógenos, entre otros).

\section{Diseño experimental}

Se realizó un diseño experimental completamente aleatorizado (DCA) balanceado, con efecto interactivo del tiempo, donde se evaluaron 2 tratamientos con tres repeticiones $(2 \times 3)$. Se adecuaron 6 unidades experimentales las cuales consistían de acuarios de 40 I sin biofiltro, con un suministró de aireación constante (aireador de $2500 \mathrm{cc} / \mathrm{min}$ ). Se evaluaron dos densidades de siembra durante 42 días; tratamiento 1 (30 individuos) y tratamiento 2 (60 individuos). Cada tres días se realizaba un recambio total del agua en las unidades experimentales con el fin de evitar que el NAT superara el rango de tolerancia reportado para la especie (mayor de $2.2 \mathrm{mg} / \mathrm{l}$ ) (Timmonsy Ebeling, 2010a).

Cada tres días se registraba medidas de NAT y oxígeno disuelto (Kit de tests $\mathrm{Hach}^{\circledR}$ ) y semanalmente dureza general y de carbonatos (Kit de test Tetra ${ }^{\circledR}$ ), pH y temperatura (Sonda Hanna ${ }^{\circledR}$ Modelo HI 991001). Quincenalmente se capturaban 20 individuos por repetición (120 en total por tratamiento) a los cuales se les registraba algunas variables de crecimiento: peso (Balanza Vibra, Modelo DJ 820E), longitud total y estándar (calibrador vernier). Los peces se alimentaron con Truchina ${ }^{\circledR} 45 \%$ tres veces al día (08:00, 12:00, 16:00) ajustada al 10\% de la biomasa calculada a partir de los datos de peso (Ramírez-Sánchez et al., 2011).

Se determinaron algunos parámetros de productividad en los alevinos de C. carpio: Tasa de crecimiento absoluto (TCA) (Salazar y Ocampo, 2002), Ganancia de peso (GP), Tasa de crecimiento específico (TCE),Factor de condición (K) y Sobrevivencia (S) (Aguilar et al, 2010).

\section{Análisis de Datos}

Se realizó una prueba de Shapiro-Wilk para validar los supuestos de normalidad, posteriormente se realizó un ANOVA y una prueba de Tukey-Kramer para determinar diferencias en las variables de crecimiento (peso, longitud total y estándar) entre los tratamientos evaluados. Los parámetros fisicoquímicos, productivos y tasa de excreción de NAT fueron analizados con la prueba de $F$, para evaluar la homogeneidad de varianzas $y$, una prueba de t-student. Se consideró una probabilidad del $5 \%(P<0.05)$ como diferencia significativa entre los tratamientos. Para el análisis de los datos se utilizó el programa de libre distribución R-Project versión 2.9.0.

\section{Resultados}

Las variables fisicoquímicas (tabla 1) no mostraron diferencias $(P>0.05)$ entre los tratamientos ( T1 
Tabla 1. Valor promedio de los parámetros fisicoquímicos de T1 y T2. Cada valor representa el promedio, \pm la desviación estándar.

\begin{tabular}{|l|c|c|}
\hline \multicolumn{1}{|c|}{ Parámetro } & T1 & T2 \\
\hline Temperatura $\left({ }^{\circ} \mathrm{C}.\right)$ & $17.15 \pm 0.83^{\mathrm{a}}$ & $17.29 \pm 1.51^{\mathrm{a}}$ \\
\hline $\mathrm{pH}$ & $7.13 \pm 0.14^{\mathrm{a}}$ & $7.04 \pm 0.17^{\mathrm{a}}$ \\
\hline Oxígeno $(\mathrm{mg} / \mathrm{l})$ & $5.72 \pm 0.82^{\mathrm{a}}$ & $4.55 \pm 1.54^{\mathrm{a}}$ \\
\hline $\mathrm{GH}(\mathrm{mg} / \mathrm{l})$ & $17.8^{\mathrm{a}}$ & $17.8 \mathrm{a}$ \\
\hline $\mathrm{KH}(\mathrm{mg} / \mathrm{l})$ & 0 & 0 \\
\hline NAT $(\mathrm{mg} / \mathrm{l})$ & $1.24 \pm 0.10^{\mathrm{a}}$ & $1,43 \pm 0.19^{\mathrm{b}}$ \\
\hline
\end{tabular}

Las letras superíndices diferentes muestran diferencias significativas $(\mathrm{P}<0.05) . \mathrm{GH}$ : Dureza general. $\mathrm{KH}$ : Alcalinidad. NAT: Nitrógeno amoniacal total.

y T2) manteniendo las condiciones ambientales para los individuos, como la temperatura del agua $\left(\mathrm{T} 1: 17.15 \pm 0.83^{\circ} \mathrm{C}\right.$ y $\left.\mathrm{T} 2: 17.29 \pm 1.51^{\circ} \mathrm{C}\right)$ y el $\mathrm{pH}(\mathrm{T} 1$ : $7.13 \pm 0.14$ y $\mathrm{T} 2: 7.04 \pm 0.17)$.

Para el crecimiento de C. carpio, el peso promedio (tabla 2) del T1 presentó diferencias significativas $(\mathrm{P}<$ 0.05) durante los cuatro muestreos realizados. Mientras que el T2 mostró un aumento significativo $(\mathrm{P}<$ 0.05 ) entre los tres primeros muestreos correspondientes a los días 0,15 y 30, pero no hubo diferencias ( $P$ > $0.05)$ entre el día 30 y el día 42. Diferencias significativas $(\mathrm{P}<0.05)$ en longitud estándar y total (tabla 2$)(\mathrm{P}<$ 0.05 ) se obtuvieron entre los días 0,15 y 30 , pero los datos obtenidos en el día 42 son iguales a los del día 30. Sin embargo, el T2 mostró diferencias significativas $(\mathrm{P}<0.05)$ entre los dos primeros muestreos, pero los últimos tres son estadísticamente iguales $(P>0.05)$. Además, el crecimiento de los individuos fue similar ( $\mathrm{P}$ $>0.05$ ) entre los tratamientos.

Por otro lado, se calcularon los parámetros de productividad para los dos tratamientos (tabla 3).No se presentaron diferencias significativas $(P>0.05)$ entre los tratamientos. Donde se observó una alta sobrevivencia (T1: $98.8 \pm 0.90 \%$ y $\mathrm{T} 2: 97.9 \pm 0.89 \%)$.

Las tasas de excreción de NAT (Tabla 4) fueron registradas cada 15 días, presentando diferencias significativas $(P<0.05)$ entre $T 1$ y $T 2$ en todos los tres periodos evaluados.

Lo anterior, puede ser contrastado en la figura 1, donde se observa que la excreción del NAT es diferente entre los tratamientos, puesto que la pendiente $(\mathrm{m})$ de $\mathrm{T} 1$ es de 0.79 , mientras que en $\mathrm{T} 2 \mathrm{~m}$ es de 0.56 , teniendo en cuenta que T2 tiene el doble de biomasa respecto de $\mathrm{T} 1$.

\section{Discusión}

Los parámetros fisicoquímicos en los sistemas acuícolas determinan el estado de salud de los peces afectando el crecimiento, la reproducción y sobrevivencia

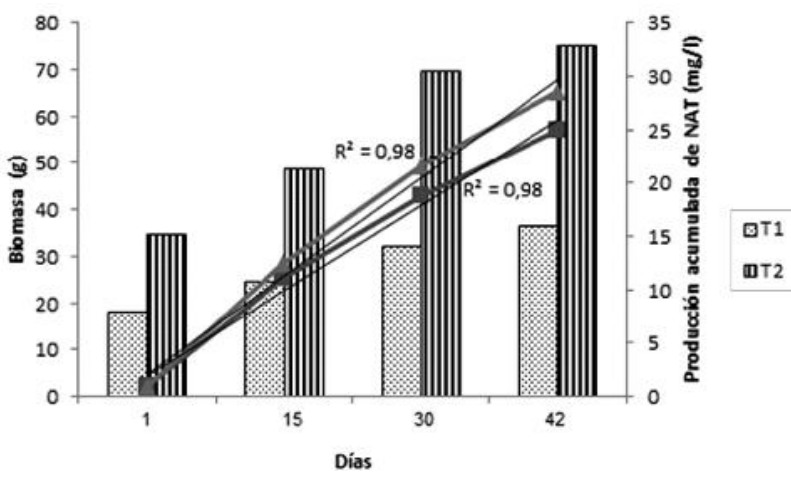

Figura 1. Valor promedio de la biomasa de C. carpio en relación a la producción acumulada de amonio de los tratamientos. Cada valor representa el promedio, \pm la desviación estándar de la biomasa para las tres repeticiones. Los cuadros (T1) y los triángulos (T2) muestran la excreción de amonio acumulada.

Tabla 2. Valor promedio de variables de crecimiento de alevinos de C. carpio, en T1 y T2, en diferentes muestreos. Cada valor representa el promedio, \pm la desviación estándar.

\begin{tabular}{|c|c|c|c|c|c|c|}
\hline \multirow{2}{*}{ Día } & \multicolumn{2}{|c|}{ Peso $(\mathbf{g})$} & \multicolumn{2}{c|}{ Longitud Estándar $(\mathbf{c m})$} & \multicolumn{2}{c|}{ Longitud Total $(\mathbf{c m})$} \\
\cline { 2 - 7 } & $\mathbf{T 1}$ & T2 & T1 & T2 & T1 & T2 \\
\hline 0 & $0.61 \pm 0.01^{\mathrm{a}}$ & $0.58 \pm 0.01^{\mathrm{a}}$ & $2.57 \pm 0.04^{\mathrm{a}}$ & $2.58 \pm 0.02^{\mathrm{a}}$ & $3.33 \pm 0.05^{\mathrm{a}}$ & $3.28 \pm 0.03^{\mathrm{a}}$ \\
\hline 15 & $0.83 \pm 0.02^{\mathrm{b}}$ & $0.81 \pm 0.05^{\mathrm{b}}$ & $2.89 \pm 0.06^{\mathrm{b}}$ & $2.97 \pm 0.08^{\mathrm{b}}$ & $3.72 \pm 0.09^{\mathrm{b}}$ & $3.74 \pm 0.12^{\mathrm{b}}$ \\
\hline 30 & $1.08 \pm 0.06^{\mathrm{c}}$ & $1.18 \pm 010^{\mathrm{c}}$ & $3.20 \pm 0.17^{\mathrm{c}}$ & $3.16 \pm 0.13^{\mathrm{b}}$ & $3.96 \pm 0.06^{\mathrm{c}}$ & $3.96 \pm 0.13^{\mathrm{b}}$ \\
\hline 42 & $1.23 \pm 0.06^{\mathrm{d}}$ & $1.27 \pm 0.12^{\mathrm{c}}$ & $3.08 \pm 0.01^{\mathrm{c}}$ & $3.14 \pm 0.10^{\mathrm{b}}$ & $3.87 \pm 0.01^{\mathrm{c}}$ & $3.92 \pm 0.11^{\mathrm{c}}$ \\
\hline
\end{tabular}

Filas con letras superíndices diferentes dentro de cada parámetro indican diferencias significativas entre los muestreos $(P<0.05)$. Entre los tratamientos no hubo diferencias significativas $(P>0.05)$ por lo que no se asignaron letras. 
Tabla 3. Valor promedio de los parámetros de productividad en $\mathrm{T} 1$ y $\mathrm{T} 2$. Cada valor representa el promedio, \pm la desviación estándar.

\begin{tabular}{|l|c|c|}
\hline \multicolumn{1}{|c|}{ Parámetro } & $\begin{array}{c}\text { T1 } \\
\mathbf{3 0} \text { peces } \\
\text { Densidad: } \\
\mathbf{0 . 4 5} \pm \mathbf{0 . 1 0} \mathbf{g} / \mathbf{l}\end{array}$ & $\begin{array}{c}\text { T2 } \\
\mathbf{6 0} \text { Peces } \\
\text { Densidad: } \\
\mathbf{0 . 8 6} \pm \mathbf{0 . 1 8} \mathbf{g} / \mathbf{I}\end{array}$ \\
\hline Peso inicial (g) & $0.61 \pm 0.14^{\mathrm{a}}$ & $0.57 \pm 0.12^{\mathrm{a}}$ \\
\hline Peso final (g) & $1.23 \pm 0.40^{\mathrm{a}}$ & $1.27 \pm 0.39^{\mathrm{a}}$ \\
\hline $\begin{array}{l}\text { Biomasa } \\
\text { inicial }(\mathrm{g})\end{array}$ & $18.33 \pm 0,13^{\mathrm{a}}$ & $34,66 \pm 0,33^{\mathrm{b}}$ \\
\hline Biomasa final $(\mathrm{g})$ & $36.92 \pm 1.71^{\mathrm{a}}$ & $75.01 \pm 6.55^{\mathrm{b}}$ \\
\hline GP/individuo/día & $2.42 \pm 0.05 \%^{\mathrm{a}}$ & $2.86 \pm 0.11 \%^{\mathrm{a}}$ \\
\hline K & $3.86 \pm 0.01^{\mathrm{a}}$ & $3.91 \pm 0.10^{\mathrm{a}}$ \\
\hline TCA & $0.01^{\mathrm{a}}$ & $0.01^{\mathrm{a}}$ \\
\hline TCE & $1.67 \pm 0.0 \%^{\mathrm{a}}$ & $1.87 \pm 0.07 \%^{\mathrm{a}}$ \\
\hline S & $98.8 \pm 0.9 \%^{\mathrm{a}}$ & $97.9 \pm 0.89 \%^{\mathrm{a}}$ \\
\hline
\end{tabular}

Las letras superíndices diferentes representan diferencias significativas entre los tratamientos $(P<0.05)$.

Tabla 4. Valores de la tasa de excreción de NAT, en los tratamientos a diferentes periodos. Cada valor representa el promedio, \pm la desviación estándar.

\begin{tabular}{|c|c|c|}
\hline \multirow{2}{*}{ Día } & \multicolumn{2}{|c|}{$\begin{array}{c}\text { Tasa de excreción de NAT } \\
(\boldsymbol{\mu g} \text { g-1 hora-1) }\end{array}$} \\
\cline { 2 - 3 } & $\mathbf{T 1}$ & $\mathbf{T 2}$ \\
\hline 14 & $0.69 \pm 0.009^{\mathrm{a}}$ & $0.40 \pm 0.02^{\mathrm{b}}$ \\
\hline 28 & $0.53 \pm 0.02^{\mathrm{a}}$ & $0.28 \pm 0.01^{\mathrm{b}}$ \\
\hline 42 & $0.47 \pm 0.02^{\mathrm{a}}$ & $0.26 \pm 0.02^{\mathrm{b}}$ \\
\hline
\end{tabular}

Las letras superíndices representan diferencias significativas $(P<0.05)$ entre los tratamientos.

de los mismos, definiendo en buena parte el éxito del cultivo (Timmons y Ebeling, 2010a). Razón por la cual, su continuo análisis y seguimiento es de importancia para el adecuado crecimiento de los individuos, manteniendo las condiciones entre los rangos de tolerancia reportados para C. carpio (Aguirre y Poss, 2000; Corantioquia, 2006; FAO, 2015a) para ambos tratamientos. Lo cual posiblemente permitió que los individuos de T1 y T2 no mostrarán diferencias significativas $(P>0.05)$ en las variables de crecimiento y parámetros productivos (factor de condición K, TCA, TCE, GP y S), similar a lo obtenido por Damsgårda et al. (2011) que no encontró diferencias en el crecimiento de los peces en relación a la densidad de siembra, trabajando con Paralichthys californicus (Merino el al., 2007),

El crecimiento durante el tiempo mostró diferencias significativas $(\mathrm{P}<0.05)$. Además, se encontró una relación inversamente proporcional entre el peso corporal o tamaño y la tasa de excreción de NAT. Este cambio metabólico, ha sido descrito para mamíferos (White y Seymour, 2005) y peces (Cech y Brauner, 2011), donde la tasa metabólica es dependiente del tamaño corporal en relación inversa, generando así una tasa de excreción de NAT relativamente menor con respecto al tiempo (Ip et al., 2001; Freedman y Noakes, 2002). Específicamente para la excreción de NAT ValvuenaVillareal y Vásquez-Torres (2011) reportaron en Oreochromis sp una disminución en la tasa de excreción al incrementar la masa corporal, manteniendo esta dinámica al cambiar la temperatura o el nivel de proteína en la dieta.

Por otro lado, la relación entre la densidad de siembra, el crecimiento, la sobrevivencia, la calidad del agua y en especial los productos nitrogenados, son factores ambientales que pueden actuar como elementos de estrés para los organismos acuáticos, en ambientes naturales y artificiales (Sumagaysay-Chavoso y San Diego-McGlone, 2003; Bo et al., 2010). Específicamente la concentración NAT y la densidad, son factores importantes, que en general han mostrado correlaciones positivas entre ellas (Ford y Langdom, 2000; Biswas et al., 2006). Se encontró que al aumentar la densidad, la concentración de NAT en el agua fue mayor, dicho comportamiento ya había sido reportado en otras especies como: Haliotis tuberculata (Mgaya y Mercer, 1995), Ctenopharyngodon idella (Sharma y Sharma, 1998), Haliotis asinina (Capinpin et al., 1999), Haliotis rubra (Huchette et al., 2003), Cyprinus carpio (Biswas et al., 2006), en el hibrido Morone saxatilis x Morone chrysops (Liu et al., 2009) Gadus morhua (Damsgårda et al., 2011), permitiendo la determinación de densidades adecuadas para la siembra sin afectar su desarrollo (Bo et al., 2010).

De acuerdo a lo anterior, la concentración de NAT en el agua fue más alta en el T2, aunque la tasa de excreción fue mayor $(P<0.05)$ en el T1. Esto podría ser explicado en relación a la capacidad que tienen los peces y otros organismos acuáticos de modificar sus tasas de excreción por cambios de presión parcial dependiendo de la concentración de NAT en el medio (Evans et al., 2005), este mecanismo de defensa puede estar acompañado por una disminución en la producción de NAT endógeno (Lim et al., 2001; Lim et al., 2004; Ip et al., 2005; Randall y Ip, 2006) causada posiblemente por una menor ingesta de alimento alterando así tasas 
de degradación y producción de aminoácidos (Wicks y Randall, 2002; Schram et al., 2010).

Teniendo en cuenta que $C$. carpio es un pez de consumo de gran importancia a nivel mundial (FAO, 2015b) $y$ es de las pocas especies de consumo que se pueden cultivar sin calefacción en la Sabana de Bogotá, es importante controlar los productos nitrogenados, en especial en sistemas cerrados de recirculación y acuapónicos. En sistemas de recirculación diseñados a mínimo costo, como los reportados por MartínezMoreno et al. (2011) y Carrascal (2011), los cuales tenían biofiltros simples, donde la capacidad de convertir los compuestos nitrogenados fue relativamente baja, la densidad aproximada de peces que se podría mantener teniendo en cuenta las condiciones experimentales de este estudio sería de: $35.4 \mathrm{~kg} / \mathrm{m}^{3}$ o 58000 peces $/ \mathrm{m}^{3}$. Estos datos también permitieron estimar y alcanzar biomasas superiores a los $10 \mathrm{~kg} / \mathrm{m}^{3}$ en sistemas acuapónicos, como se demostró en el trabajo de goldfish-carpa-orégano de Cifuentes-Torres y TorresMesa (2012).

En conclusión se observaron tres patrones que relacionan la excreción de NAT y la densidad, donde se obtuvo un aumento lineal de NAT acumulado durante el tiempo de estudio, aunque las tasas de excreción variaron con respecto al tiempo, por otro lado, se halló una relación inversa entre la densidad y la tasa de excreción de NAT, pues al aumentar la densidad, se disminuyó la tasa excreción.

\section{Agradecimientos}

A la Universidad Militar Nueva Granada, su vicerrectoría de Investigaciones y a Colciencias por el proyecto CIAS 585.

\section{Referencias}

Aguilar F, Afanador-Téllez G, Muñoz-Ramírez A. Efecto del procesamiento de la dieta sobre el desempeño productivo de tilapia nilótica (Oreochromis niloticus Var. Chitralada) en un ciclo comercial de producción. Revista de la Facultad de Medicina Veterinaria y de Zootecnia. 2010;57(2):104-118.

Aguirre W, Poss SG. Non-native aquatic species in the gulf of Mexico and south atlantic regions. Gulf status marine fisheries commission 2000; [15 de Abril del 2009] URL: http://nis.gsmfc.org/.

Ballantyne JS. 2001. Amino acid metabolism. En: Wright P, Anderson $\mathrm{P}$ (Editores). Nitrogen excretion. Editorial Academia Press. EEUU. p. 77 - 108.

Biswas JK, Sarkar D, Chakraborty P, Bhakta JN, Jana BB. Density dependent ambient ammonium as the key factor for optimization of stocking density of common carp in small holding tanks. Aquaculture. 2006;261:952-300.
Bo G, Wei H, Tang Lin Z, Ming D, Deliang L, Ya Ping W, Zuo Yan Z. Acute and chronic un-ionized ammonia toxicity to 'all-fish' growth hormone transgenic common carp (Cyprinus carpio L.). Hydrobiology. 2010; 55:4032-4037.

Carrascal JR. 2011. Evaluación de la etapa de levante de carpa común (Cyprinus carpio) en sistemas cerrados de recirculación de agua. Trabajo de grado para optar al título de biólogo. Universidad Militar Nueva Granada Facultad de Ciencias Programa de Biología Aplicada. 58 p.

Capinpin EC, Toledo JD, Encena VC, Doi M. Density dependent growth of the tropical abalone Haliotis asinina in cage culture. Aquaculture. 1999;171: 227-235.

CCAC. Guidelines on: the care and use of fish in research, teaching and testing.Canadian Council on Animal Care 2005; [21 de mayo del 2015] URL: http://www.ccac.ca/Documents/Standards/Guidelines/Fish.pdf.

Cech JJ, Brauner CJ. 2011. Techniques in Whole Animal Respiratory Physiology. En: Farrel AP (Editor). Encyclopedia of fish physiology: from genome to environment. Academic Press, Inglaterra, p. $846-863$.

Cifuentes-Torres ML, Torres-Mesa AC. Evaluación del crecimiento de goldfish (Carassius auratus), carpa (Cyprinus carpio) y orégano (Origanum vulgare) en un Sistema Acuapónico 2012; [30 de Enero del 2013] URL: http://hdl.handle.net/10654/6530.

Corantioquia -Coorporación Autónoma Regional. 2006. Carta de Acuicultura. Medellín, Colombia.

Chakraborty B, Mirza M. Effect of stocking density on survival and growth of endangered bata, Labeo bata (Hamilton-Buchanan) in nursery ponds. Aquaculture. 2007;265:156-162.

Damsgårda B, Bjørklund F, Johnsen HK, Toften H. Short- and longterm effects of fish density and specific water flow on the welfare of Atlantic cod, Gadus morhua. Aquaculture. 2011;322-323: 184 - 190 .

Evans DH, Piermarini PM, Potts WTW. Ionic transport in the fish gill epithelium. J Exp Zool. 1999;283:641 - 652.

FAO. Cultured aquatic species information programme 2015a; [22 Mayo de 2015] URL: http://www.fao.org/fishery/culturedspecies/Cyprinus_carpio/en.

FAO. Colecciones de estadísticas de pesca: Producción mundial 2015b; [21de mayo de 2015] URL: http://www.fao.org/fishery/statistics/global-production/es.

Ford E, Langdon CJ. Co-culture of Dulse Palmaria mollis and Red Abalone Haliotis rufescensunder limited flow conditions. Aquaculture. 2000; 185:137-158.

Freedman JA, Noakes DLG. Why are there no really big bony fishes? a point-of-view on maximun body size in teleosts and elasmobranchs. Kluwer Academic Publishers. 2002;12:403-416.

González EM, Pedraza A, Pérez MM. Caracterización agrológica del suelo y diagnóstico de su fertilidad en la estación experimental del Campus Nueva Granada, Cajicá (Cundinamarca, Colombia). Revista Facultad de Ciencias Básicas. 2009;4(1):82-104. 
Gobernación de Cundinamarca. Plan Integral Unico 2011; [21 de mayo de 2015] URL: http://www1.cundinamarca.gov.co/ PIU-2012/CUNDINAMARCA\%20\%202012/PLANES\%20 INTEGRALES\%20\%C3\%9ANICOS\%20-\%20PIU/DOCUMENTOS\%2OPIU/CUNDINAMARCA_CAIICA/PIU.pdf.

Hargreaves JA, Tucker CS. 2004. Industry development. En: Tucker CS,Hargreaves JA (Editores). Biology and culture of channel catfish. Elsevier.Amsterdam, Alemania, p. 1-14

Huchette S, Koh C, Day RW. Growth of juvenile blacklip abalone (Haliotis rubra) in aquaculture tanks: Effects of density and ammonia. Aquaculture. 2003;219: 457-470.

Ip YK, Chew SF, Randall DJ. 2001. Ammonia toxicity, tolerant and excretion. En: Wright P, Anderson P (Editores). Nitrogen excretion. Academia Press, EEUU,p. 109-148.

Ip YK, Chew SF, Wilson JM, Randall DJ. Defences against ammonia toxicity in tropical air-breatihng fishes exposed to high concentrations of environmental ammonia: a review. J Comp Physiol B. 2004;174: 565 - 575 .

Ip YK, Leong MWF, Sim MY, Goh GS, Chew SF. Chronic and acute ammonia toxicity in mudskippers, Periophthalmodon schlosseri and Boleophthalmus boddaerti: brain ammonia and glutamine contents, and effects of methionine sulfoximine and MK801. J Exp Biol. 2005;208:1993-2004.

Ip YK, Chew SF. Ammonia production, excretion, toxicity and defense in fish: a review. Front Physiol. 2010; 1:1-20.

Kaushik SJ. Factores que afectan la excreción nitrogenada en teleósteos y crustáceos. 2000. En: Civera-Cerecedo R, Pérez-Estrada CJ, Ricque-Marie D, Cruz-Suárez LE (Editores). Avances en nutrición acuícola memorias del IV Simposium internacional de nutrición acuícola. Monterrey, México, p.237-250.

Lim CB, Anderson PM, Chew SF, Ip YK. Reduction in the rates of protein and amino acid catabolism to slow down the accumulation of endogenous ammonia: a strategy potentially adopted by mudskippers (Periophthalmodon schlosseri and Boleophthalmus boddaerti) during aerial exposure in constant darkness. J Exp Biol. 2001;204:1605-1614.

Lim CK, Chew SF, Tay ASL, Ip YK. Effects of peritoneal injection of $\mathrm{NH}_{4} \mathrm{HCO}_{3}$ on nitrogen excretion and metabolism in the swamp eel Monopterus albus increased ammonia excretion with an induction of glutamine synthetase activity. J Exp Zool. 2004;301a: 324-333.

Liu FG, Yang SD, Chen HC. Effect of temperature, stocking density and fish size on the ammonia excretion in palmetto bass (Morone saxatilis x M. chrysops). Aquacult Res. 2009;40:450 - 455.

Martínez-Moreno O, Gómez-Ramírez E, Hurtado-Giraldo H. Levante de Goldfish (Carassius auratus) en sistemas de recirculación cerrado. Revista Facultad de Ciencias Básicas. 2011;7(2): 260273.

Mercado Burgos I, García JL, Rosado Cárcamo R, Olaya-Nieto CW, Segura Guevara FF, Brú Cordero SB, Tordecilla-Petro G. Cultivo de dorada (Brycon sinuensis Dahl, 1955) en jaulas flotantes a diferentes niveles de proteína. Rev Colomb Cienc Pecu. 2006;19:204-211.
Merino GE, Piedrahita RH, Conklin DE. The effect of fish stocking density on the growth of California halibut (Paralichthys californicus) juveniles. Aquaculture. 2007;265:176-186.

Mgaya YD, Mercer JP. The effects of size grading and stocking density on growth performance of juvenile abalone, Haliotis tuberculata Linnaeus. Aquaculture. 1995;136:297-312.

Papoutsoglou SE, Papoutsoglou E, Alexis MN. Effect of density on growth rate and production of rainbow trout(Salmo gairdtteriRich.) Over a full rearing period. Aquaculture. 1987;66:9-17.

Rahman MA, Mazid MA, Rahman MR, Kahan MN, Hossain MA, Hussain MG. Effect of stocking density on survival and growth of critically endangered mahseer, Tor putitora (Hamilton), in nursery ponds. Aquaculture. 2005;249:275 - 284.

Ramírez-Sánchez LM, Pérez-Trujillo MM, Jimenez P, Hurtado-Giraldo H, Gómez-Ramírez E. Evaluación preliminary de sistemas acuapónicos e hidropónicos en cama flotante para el cultivo de orégano (Origanum vulgare: Lamiaceae). Revista Facultad de Ciencias Básicas. 2011;7(2):242-259.

Randall DJ, Ip YK. Ammonia as a respiratory gas in water and airbreathing fishes. Respir Physiol Neurobiol. 2006;154:216-225.

Reddy-Lopata K, Auerwald L, Cook P. Ammonia toxicity and its effect on the growth of the South Africa abalone Haliotis midae Linnaeus. Aquaculture. 2006; 261:678-687.

Remen M, Imsland AK, Stefansson SO, Jonassen TM, Foss A. Interactive effects of ammonia and oxigen on growth and physiological status of juvenil atlantic cod (Gadus morhua). Aquaculture. 2008;274:292-300.

Rombough PJ. 2011. Respiratory gas exchange during development: models and mechanisms. En: Farrel AP (Editor). Encyclopedia of fish physiology: from genome to environment. Academic Press, Inglaterra, p. 829 - 837.

Salazar MB, Ocampo D. Tasa de crecimiento del pez ángel Pterophyllum scalre (Perciformes: Chiclidae) en condiciones de laboratorio. Acta Universitaria. 2002; 12:28-33.

Schram E, Roques JAC, Abbink W, Spannings T, de Vries P, Bierman $\mathrm{S}$, van de Vis $\mathrm{H}$, Flick $\mathrm{G}$. The impact of elevated water ammonia concentration on physiology, growth and feed intake of african catfish (Clarias gariepinus). Aquaculture. 2010;306:108-115.

Scott DM, Lucas MC, Wilson RW. The effect of high ph onion balance, nitrogen excretion and behaviour in freshwater fish from an eutrophic lake: a laboratory and field study. Aquatic Toxicology. 2005;73:31-43.

Sharma JG, Sharma R. Effects of different stocking densities on survival and growth of grass carp, Ctenopharyngodon idella, larvae using a recirculating culture system. J. Appl. Aquacult. 1998;8:79-83.

Sinha AK, Liew HJ, Diricx M, Blust R, De Boeck G. The interactive effects of ammonia exposure, nutritional status and exercise on metabolic and physiological responses in goldfish (Carassius auratus L.). Aquatic Toxicology. 2012a;109:33-46.

Sinha AK, Diricx M, Chan LP, Liew HJ, Kumar V, Blust R, De Boeck G. Expression pattern of potential biomarker genes related to growth, ion regulation and stress in response to 
ammonia exposure, food deprivation and exercise in common carp (Cyprinus carpio). Aquatic Toxicology. 2012b;122123: 93-105.

Sumagaysay-Chavoso NS, San Diego-McGlone ML. Water quality and holding capacity of intensive and semi-intensive milkfish (Chanos chanos) ponds. Aquaculture. 2003;219: 413-429.

Timmons MB, Ebeling JM. 2010a. Water Quality. En: Timmons MB, Ebeling JM (Editores). Recirculating Aquaculture. Cayuga Aqua Ventures. Ithaca, EEUU, p. 39-78.

Valderrama M, Valderrama E, González JD. La Pesca en la laguna de Fúquene: una aproximación a su situación actual y sus perspectivas de manejo. Instituto Humboldt-Colombia 2004; [05 de Enero del 2011]. URL:http://www.ibcperu.org/doc/ isis/8779.pdf.

Valvuena-Villareal RD, Vásquez-Torres W. El peso corporal esta inversamente relacionado con la excreción de amonio en tilapia roja (Oreochromis sp). Rev Colomb Cienc Pecu. 2011;24(2):191-200.

Walsh PJ, Mommsen TP. 2001. Evolutionary considerations of nitrogen metabolism and excretion.En: Wright P, Anderson P (Editores). Nitrogen Excretion.Academic Press. San Diego, EEUU, p.1-30.

White CR, Seymour RS. Allometric scaling of mammalian metabolism. The J Exp Biol. 2005;206:1611-1619.

Wicks BJ, Randall DJ. The effect of feeding and fasting on ammonia toxicity in juvenile rainbow trout, Oncorhynchus mykiss. Aquaic Toxicology. 2002;59:71-82.

Wood CM. 2001. Influence of feeding, exercise and temperature on nitrogen metabolism and excretion. En: Wright P, Anderson P (Editores). Nitrogen Excretion. Academia Press.San Diego, EEUU, p. 201 - 238. 